Draft version September 29, 2018

Preprint typeset using $\mathrm{IAT}_{\mathrm{E}} \mathrm{X}$ style emulateapj v. 5/2/11

\title{
THEORETICAL EXPLANATION OF THE COSMIC RAY PERPENDICULAR DIFFUSION COEFFICIENT IN THE NEARBY STARBURST GALAXY NGC 253
}

\author{
K. Buffie ${ }^{1}$, V. Heesen ${ }^{2}$, And A. Shalchi ${ }^{1}$ \\ ${ }^{1}$ Department of Physics and Astronomy, University of Manitoba, Winnipeg, Manitoba R3T 2N2, Canada, shalchi@physics.umanitoba.ca \\ and \\ ${ }^{2}$ School for Physics and Astronomy, University of Southampton, Southampton SO17 1BJ, UK, v.heesen@soton.ac.uk \\ Draft version September 29, 2018
}

\begin{abstract}
Diffusion coefficients are usually used to describe the propagation of Cosmic Rays through the Universe. Whereas such transport parameters can be obtained from experiments in the Solar System, it is difficult to determine diffusion coefficients in the Milky Way or in external galaxies. Recently a value for the perpendicular diffusion coefficient in the nearby starburst halaxy NGC 253 has been proposed. In the present paper we reproduce this value theoretically by using an advanced analytical theory for perpendicular diffusion.

Subject headings: cosmic rays - galaxies: magnetic fields - galaxies: individual (NGC 253) - galaxies: starburst
\end{abstract}

\section{INTRODUCTION}

Radio continuum observations of the nearby starburst galaxy NGC 253 can be used to measure the distribution and transport of Cosmic Ray electrons (see Heesen et al. 2009a, 2011). The current understanding is that Cosmic Ray electrons are accelerated in the disk by shock waves induced by supernova explosions (Revnolds et al. 2012). The electrons are then transported away over their lifetime by either convection in a galactic wind or diffusion, where the two processes can be distinguished from their different typical transport length as a function of electron energy. The magnetic field in galaxies is normally dominated by the turbulent component, but averaged over spatial sizes larger than $1 \mathrm{kp} 1$ (a typical resolution achieved with radio interferometers in external galaxies) ordered magnetic field components are detected by their linear polarisation from synchroton emission of Cosmic Ray electrons spiralling in the magnetic field. Observations suggest that galaxies in their disk plane are dominated by a magnetic field that is parallel to the disk and has a spiral pattern with a constant pitch angle of a few tens of degrees (Beck 2012). Further away from the disk plane at heights larger than $1 \mathrm{kpc}$ the field becomes more vertical and the field lines open up further into the halo (Haverkorn \& Heesen 2012).

The motion of Cosmic Rays through the interplanetary or interstellar space is complicated due to their interaction with turbulent magnetic fields. Such fields lead to spatial diffusion of the energetic particles. In addition to the turbulent magnetic fields one can also observe a non-vanishing mean magnetic field which is in the case of the interstellar medium the Galactic magnetic field. This mean field breaks the symmetry of the physical system and one has to distinguish between diffusion along and across the Galactic magnetic field. The former process is also known as parallel diffusion whereas the latter process is called perpendicular transport. The theoretical investigation of Cosmic Ray transport has a

\footnotetext{
${ }^{1}$ In the present article we measure distances in parsecs (pc). Approximately we have $1 \mathrm{pc} \approx 3 \cdot 10^{16} \mathrm{~m} \approx 3.31 \mathrm{y}$.
}

long history (see, e.g., Schlickeiser 2002). Whereas the propagation of cosmic particles through the solar system seems to be well understood (see, e.g., Shalchi et al. 2006; (Tautz \& Shalchi 2012), it is unclear whether advanced transport theories can describe particle propagation with high accuracy in other systems like the Milky Way or external galaxies. At least parallel diffusion seems to be a process which can be described very well by more advanced diffusion theories. The measured decrease of the abundance ratio of secondary to primary Cosmic Ray nuclei as B/C and N/O at kinetic energies above $1 \mathrm{GeV} /$ nucleon, implies a variation of the total column density as a function of rigidity $R$ as (Swordy et al. 1990) $\lambda_{\|} \propto R^{0.6}$ where we have used the parallel mean free path $\lambda_{\|}$. This behavior was explained by Shalchi \& Schlickeiser (2005) who used a nonlinear diffusion theory. The behavior of low energy Cosmic Rays was also explained by using an extension of quasilinear theory (see Shalchi \& Büsching 2010).

More complicated, however, is the process of perpendicular diffusion. In the recent years progress has been achieved. Shalchi (2010) developed an advanced theory for perpendicular diffusion which contains all known limits of diffusion theory. This theory is called the Unified Non-Linear Transport (UNLT) theory and provides the correct subdiffusive behavior for one-dimensional turbulence in agreement with the so-called theorem on reduced dimensionality (see Jokipii et al. 1993 and Jones et al. 1998) and computer simulations (see, e.g., Qin et al. 2002). Furthermore, the correct field line random walk limit can be derived in agreement with the nonlinear field line transport theory of Matthaeus et al. (1995). The theory discussed here was also tested numerically confirming its validity for solar wind parameters (see Tautz \& Shalchi 2011).

In analytical theories for energetic particle transport, the model of interstellar turbulence enters the corresponding nonlinear integral equation for the perpendicular diffusion coefficient. Shalchi et al. (2010) have therefore combined the UNLT theory with the turbulence correlation tensor proposed by Cho et al. (2002) based 
on Goldreich-Sridhar scaling (see Goldreich \& Sridhar 1995). Later, however, Heesen et al. (2011) compared the analytical result obtained in the aforementioned paper with observations of NGC 253 and noticed a major difference. Using a multi-wavelength picture of the starburst nucleus they established that the nuclear outflow of hot X-ray emitting gas can be collimated by the magnetic field lines in the walls that surround the conical outflow. The magnetic field lines are aligned with the wall orientation as would be expected from compression by the hot gas in the outflow cone. An annotated figure of the multi-wavelength picture can be found in Figure 1, which shows the approximate location of the magnetic field filaments that are the walls of the outflow cone seen in projection. We have overlaid contours of the $\lambda \mathbf{3} \mathbf{c m}$ radio continuum emission at a resolution of $150 \mathrm{pc}$ that show the extent of the filaments extending along the northwestern outflow cone. The filaments are also observed at $\lambda \lambda 20$ and $6 \mathrm{~cm}$ at the same spatial resolution, which offers to study the perpendicular diffusion coefficient as described in Section 3 .

These observations led Heesen et al. (2011) to the finding that the observed perpendicular diffusion coefficient is a factor of 10 too high in comparison to the predicted value by the theory. In the present paper we, therefore, revisit the problem of perpendicular transport of energetic particles in the nearby starbust galaxy NGC 253. We combine the approach of Shalchi et al. (2010) with more realistic particle and turbulence parameters to compute the perpendicular diffusion coefficient more accurately.

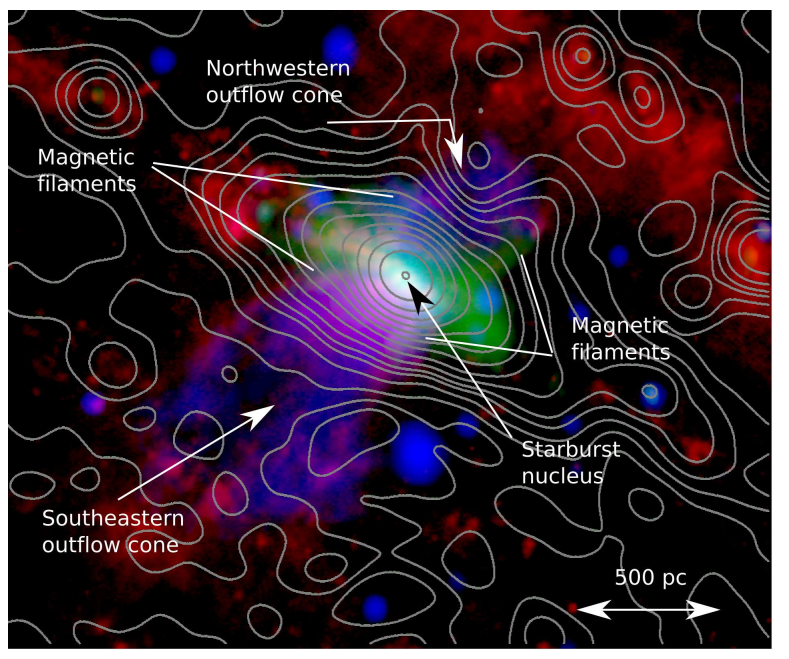

Figure 1. Three-colour composite, multi-wavelength view of the central region in NGC 253. Red, green, and blue indicate $\mathrm{H} \alpha$ from Westmoquette at al. (2011), $\lambda 20 \mathrm{~cm}$ radio continuum, and Chandra soft X-ray (M. Hardcastle, priv. com.), respectively. Contours show $\lambda 3 \mathrm{~cm}$ radio continuum at $150 \mathrm{pc}$ resolution with the first contour at $3 \times$ the r.m.s noise level and each consecutive contour increasing roughly by a factor of two. An identical figure without the annotations and contours can be found in Heesen et al. (2011).

\section{THEORETICAL DESCRIPTION OF PERPENDICULAR DIFFUSION}

The analytical description of diffusion across the mean magnetic field has a long history (see Shalchi 2009, for a review). Very recently an advanced theory for perpendicular transport has been developed (see Shalchi 2010) which shows good agreement with computer simulations performed for the parameters of the solar wind (see Tautz \& Shalchi 2011). This theory is called the Unified Non-Linear Transport (UNLT) theory and provides a nonlinear integral equation for the perpendicular diffusion coefficient $\kappa_{\perp}$. According to this equation $\kappa_{\perp}$ depends on magnetic turbulence described by the so-called magnetic correlation tensor. Therefore, the understanding of turbulence is crucial for the analytical description of cross field diffusion. A model for the magnetic correlation tensor in the interstellar medium was proposed by Cho et al. (2002) which is based on the GoldreichSridhar model (Goldreich \& Sridhar 1995). For this specific turbulence model, Shalchi et al. (2010) derived the following equation

$$
\begin{aligned}
\frac{\lambda_{\perp}}{\lambda_{\|}} & =\frac{a^{2}}{6}\left(\frac{\delta B}{B_{0}}\right)^{2 / 3} \int_{0}^{1} d y \int_{0}^{\infty} d x e^{-\left(x y^{2 / 3} E_{B}^{-4 / 3}\right)} \\
& \times \frac{2 x^{2} y^{2}+1}{x^{2} y^{2}+1} \frac{y^{7 / 3}}{x^{2} y^{4} \lambda_{\|} / \lambda_{\perp}+y^{2}+4 \lambda_{\|} \lambda_{\perp} /(3 l)^{2}}
\end{aligned}
$$

where we have used the perpendicular mean free path which is related to the perpendicular diffusion coefficient via $\lambda_{\perp}=3 \kappa_{\perp} / 3$. According to Eq. (1) the parameter $\lambda_{\perp}$ depends on the parallel mean free path $\lambda_{\|}=3 \kappa_{\|} / v$ ( $v$ is the particle speed) and, therefore, there is a mutual influence of parallel and perpendicular transport. The parameter $a^{2}$ in Eq. (1) is an order one constant related to the probability that the particle is scattered away from the magnetic field lines (see, e.g., Dosch et al. 2009). In the present paper we set $a^{2}=1$ for simplicity. The perpendicular diffusion coefficient is also controlled by the characteristic length scale of the turbulence $l$, and the ratio $E_{B} \equiv \delta B / B_{0}$. The latter ratio is a measure for the turbulent magnetic field strength $\delta B$ with respect to the mean field $B_{0}$. The mean field is $B_{0}$ defined as the spatially averaged magnetic field vector on a scale corresponding to the length $l$, which is usually related to the correlation length of magnetic turbulence. The latter parameter is a characteristic scale for the correlation of magnetic fields at two different positions in space. Often this scale is directly proportional to the so-called bendover scale which denotes the turnover from the inertial range of the turbulence to the energy range. Furthermore, this scale is smaller than the largest scales on which turbulence can be observed.

Shalchi et al. (2010) have solved Eq. (1) numerically to obtain values for the perpendicular diffusion coefficient in the interstellar medium. Heesen et al. (2011), however, compared the theoretical results with observations and found a disagreement. Below we, therefore, perform a parameter study to compute the perpendicular diffusion coefficient and compare it with observations. We will show that for the correct choice of input parameters, Eq. (11) is indeed able to reproduce the aforementioned observations.

\section{OBSERVATIONS}

The different parameters entering Eq. (10) as well is the perpendicular diffusion coefficient itself can be obtained from radio continuum observations of the nearby galaxy 
NGC 253 as described in Heesen et al. (2009a, 2011). In this section, we briefly summarize the techniques they have been using to quantify the Cosmic Ray transport.

Radio continuum studies can be used to measure the length scale of Cosmic Ray diffusion. The time scale of Cosmic Ray electrons is determined by loss processes by which the electrons are losing their energy, such as synchrotron and inverse Compton radiation. The diffusion coefficient can be then calculated by

$$
\kappa_{\mathrm{obs}}=L_{\mathrm{diff}}^{2} / \tau
$$

where $L_{\text {diff }}$ is the diffusion length scale and $\tau$ is the electron lifetime. Depending on the magnetic field structure, the observed diffusion coefficient is either along $\left(\kappa_{\|}\right)$the magnetic field or perpendicular to it $\left(\kappa_{\perp}\right)$.

Ideally, one can measure the distance to the starformation sites, where the Cosmic Rays are accelerated and injected into the interstellar medium, to obtain the transport length scale. This is for instance the case when observing galaxies in so-called edge-on geometry, where the observer is located in or close to the disk plane of the galaxy (the inclination angle is close to $90^{\circ}$ ). Cosmic Ray acceleration in supernova remnants is confined to the relatively thin disc plane, where the formation of massive stars happens. The geometry in this case is thus simple: Cosmic Rays are transported away from the star formation sites over their lifetime and their transport length scale is equal to the vertical electron scaleheight. Typical scaleheights of galaxies are $1.8 \mathrm{kpc}$ at observing wavelengths of $\lambda 6 \mathrm{~cm}$ (Krause 2009), where longer wavelengths have larger scaleheights and shorter wavelengths smaller ones. Typical Cosmic Ray electron lifetimes in the interstellar medium are between 1 and $10 \mathrm{Myr}$. This basically determines the typical parallel diffusion coefficient in the ISM, where it is assumed that the halo magnetic field is opening up from the disk parallel to a vertical direction with increasing distance from the disk. Therefore, the vertical diffusion is predominantly along the magnetic field lines hence allowing us to measure $\kappa_{\|}$. Heesen et al. (2009b) confirmed this for NGC 253 finding the characteristic X-shaped halo magnetic field with significant vertical components that is observed in a number of nearby galaxies (e.g., Soida et al. 2011).

To measure the perpendicular diffusion coefficient is more difficult, because it requires to have variations of the Cosmic Ray distribution perpendicular to the magnetic field orientation. The observed radio continuum emission is mostly smooth in the disk-halo interface and filamentary structures are rare. Numerical MHD simulations suggest that the disk halo interface is dominated by filamentary magnetic fields (Breitschwerdt et al. 2012), but line-of-sight confusion and limited spatial resolution hampers their detection. An exception are starburst galaxies, where the spatially concentrated star formation activity results in exceptionally high radio continuum surface brightness, allowing us to employ high spatial resolution $(\approx 100 \mathrm{pc})$. The spatially concentrated star formation can result into outflows of hot X-ray emitting gas in a galactic wind. The magnetic field is then concentrated and amplified by expansion of the hot gas until a pressure equilibrium is reached. This very specific geometry allowed Heesen et al. (2011) to measure the perpendicular diffusion across the magnetic field in the walls of the nuclear outflow cone in NGC 253.

The latter authors found the width of radio continuum filaments weakly dependent on frequency and thus electron age, which can be interpreted as perpendicular Cosmic Ray diffusion. We note that they were able to measure the orientation of the ordered magnetic field along the filaments as it would be expected for a compression due to the hot X-ray emitting gas outflowing from the nuclear starburst. From the observations of particles with a magnetic rigidity of $R=3 \times 10^{12}$ Volt (equivalent to an electron energy of $3 \mathrm{GeV}$ ), they found a perpendicular diffusion coefficient of $\kappa_{\perp}=(2.6 \pm 0.6) \times 10^{28} \mathrm{~cm}^{2} \mathrm{~s}^{-1}$. For the parallel diffusion coefficient one can assume $\kappa_{\|}=1.0 \times 10^{29} \mathrm{~cm}^{2} \mathrm{~s}^{-1}$ (again for an electron energy of $3 \mathrm{GeV}$ ), as measured from the diffusion of Cosmic Ray electrons from the disk into the halo along the vertical halo magnetic field (Heesen et al. 2009a). It is important to measure the diffusion coefficients at roughly the same electron energy, because they are energy dependent. We have reduced the measured diffusion coefficient of Heesen et al. (2009a) to account for a possible contribution of convection. The southwestern halo is different from the northeastern one as indicated by the dependence of the electron scaleheight on the electron lifetime and by the different amount of extra-planar gas that is more abundant in the northeastern convective halo. However, we can not rule out the contribution of convection of Cosmic Rays by the disc wind and thus have lowered the diffusion coefficient assuming that both convection and diffusion contribute equally in the southwestern halo.

Equation (1) depends on turbulence properties, namely the ratio of magnetic fields $\delta B / B_{0}$ and the scale $l$. According to Heesen et al. (2011) the equipartion estimate for the total field strength is $B_{\text {tot }}=46 \mu \mathrm{G}$. The ordered field strength $B_{\text {ord }, \perp}$ in the sky plane can be computed from the observed degree of polarization $p$ of the synchrotron emission (e.g., Beck \& Krause 2005):

$$
p=p_{0}\left(1+\frac{7}{3} q^{2}\right) /\left(1+3 q^{2}+\frac{10}{9} q^{4}\right),
$$

where $p_{0}$ is the intrinsic degree of polarization $\left(p_{0}=(3-\right.$ $3 \alpha) /(5-3 \alpha))$ and $q$ is the ratio of the isotropic turbulent field $B_{\text {turb }}$ to the ordered field $B_{\text {ord }, \perp}$ in the plane of the sky. For a non-thermal radio spectral index of $\alpha=-1$ and a polarization degree of $p=0.21$ we obtain $B_{\text {turb }}=$ $41 \mu \mathrm{G}$ and $B_{\text {ord }, \perp}=21 \mu \mathrm{G}$. For this particular case, the ordered magnetic field lies in the sky plane so that we find for the ordered magnetic field strength $B_{0}=21 \mu \mathrm{G}$. The fluctuations in the magnetic field strength are equal to the turbulent field field strength $\left(\delta B=B_{\text {turb }}\right)$ and thus we find for the the ratio $\delta B / B_{0} \approx 2$. We note that this is an upper limit as our spatial resolution of $150 \mathrm{pc}$ that was available for the polarization measurements may be not be enough to resolve the filamentary magnetic fields. High resolution $\lambda 20 \mathrm{~cm}$ maps show a filament width of only $40 \mathrm{pc}$ in radio continuum emission (see Figure 1), so that our measured ordered magnetic field strength $B_{\text {ord }}$ is only a lower limit. We have thus studied the implication of a lower value of $\delta B / B_{0}=1$ to indicate how it would change the theoretical expectation for the perpendicular diffusion coefficient.

More difficult to estimate is the scale $l$. The width of the cone walls in which the magnetic fields are confined 
is equal or less than $40 \mathrm{pc}$ (Heesen et al. 2011). This suggests that the upper value cannot be larger than about $l \approx 50-100 \mathrm{pc}$. However the value for $l$ is very uncertain and, therefore, we compute the perpendicular diffusion coefficient for a whole range of correlation lengths. Beck (2007) for instance suggested that the largest scales of turbulence are in the order of $10-100$ pc. Such largest scales, however, are not necessarily equal to the turbulence correlation scale. Actually they can be seen as the maximum of the scale $l$. Sometimes (see, e.g., Shalchi et al. 2009) it is assumed that the correlation length of interstellar turbulence is $1 \mathrm{pc}$ but it could also be shorter. Therefore we compute the perpendicular diffusion coefficient for $1 \mathrm{pc} \leq l \leq 1000 \mathrm{pc}$ to explore the values of $l$ which lead to agreement between theory and observations.

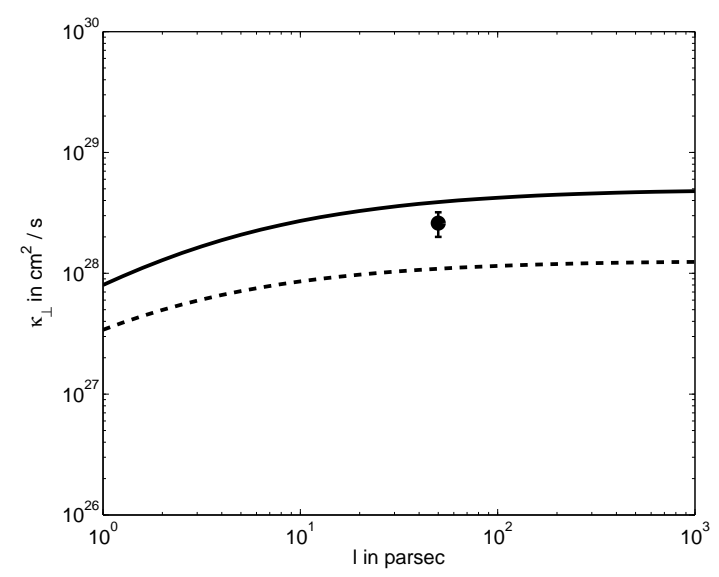

Figure 2. The perpendicular diffusion coefficients of Cosmic Rays in the nearby starbust galaxy NGC 253. We show the theoretical perpendicular diffusion coefficient for different length scales $l$. For the parallel diffusion coefficient we have used $\kappa_{\|}=$ $1.0 \times 10^{29} \mathrm{~cm}^{2} \mathrm{~s}^{-1}$ as proposed by Heesen et al. (2009a). The theoretical values were calculated for $\delta B / B_{0}=1$ (dashed line) and $\delta B / B_{0}=2$ (solid line). The dot represents diffusion coefficient from the observations (see Heesen et al. 2011), where we have $\kappa_{\perp}=(2.6 \pm 0.6) \times 10^{28} \mathrm{~cm}^{2} \mathrm{~s}^{-1}$ at $l=50 \mathrm{pc}$.

\section{REPRODUCING THE OBSERVATIONS}

In the following we solve Eq. (1) numerically for different turbulence scales $l$. To relate the mean free paths to the spatial diffusion coefficients we employ $\lambda_{\|}=3 \kappa_{\|} / v$ and $\lambda_{\perp}=3 \kappa_{\perp} / v$. In the latter relations we replace the particle speed $v$ by the speed of light $c$ since we deal with relativistic particles. As described in the previous sections we set $\kappa_{\|}=1.0 \times 10^{29} \mathrm{~cm}^{2} \mathrm{~s}^{-1}$ and $a^{2}=1$ in our equation for the perpendicular diffusion coefficient. We have calculated the latter transport parameter for $\delta B / B_{0}=1$ and $\delta B / B_{0}=2$. Our theoretical results are shown together with the observations in Fig. 2]

The observational value of the perpendicular diffusion coefficient is $(2.6 \pm 0.6) \times 10^{28} \mathrm{~cm}^{2} \mathrm{~s}^{-1}$ for a correlation length of approximately $50 \mathrm{pc}$. The theoretical perpendicular diffusion coefficient is calculated for different values of the correlation length. For a correlation length of $50 \mathrm{pc}$ we found a $\kappa_{\perp}$ which is very close to the observations depending of the value of $\delta B / B_{0}$. We note that the perpendicular diffusion coefficient is only weakly dependent on the turbulent correlation scale above a scale of approximately $10 \mathrm{pc}$. The observed diffusion coefficient seems to rule out correlation lengths smaller than $1 \mathrm{pc}$, but does not constrain very well the upper limit. It is reasonable to assume that the correlation length is smaller than the largest scales which are in the order of $10-100 \mathrm{pc}$ (see Beck 2007). We note that the observed value of the perpendicular diffusion coefficient is quite well reproduced by the measured value for $\delta B / B_{0}$, which is in the range between 1 and 2 as argued in Section 3 .

\section{SUMMARY AND CONCLUSION}

A fundamental process in astrophysics is the propagation of Cosmic Rays through the Universe. Whereas it seems that we understand the motion of energetic particles in the solar system very well (see, e.g., Shalchi et al. 2006; Tautz \& Shalchi 2012), it is still not clear how the transport parameters look like in the interstellar space of our own or external galaxies. Shalchi et al. (2010) have calculated the perpendicular diffusion coefficient for interstellar turbulence parameters. Heesen et al. (2009a, 2011) obtained measurements for the parallel and perpendicular diffusion coefficient in the nearby starbust galaxy NGC 253. They compared their results with the theoretical values presented in Shalchi (2010) and found one order of magnitude between the two results. However, the perpendicular diffusion coefficient obtained in Shalchi et al. (2010) was obtained for a different set of parameters. Especially the parallel diffusion coefficient used in the latter paper does not agree with the value obtained by the observers. Thus, in the current paper we combined the approach of Shalchi et al. (2010) with parameter sets tailored to the observations of NGC 253, such as the parallel diffusion coefficient and magnetic fields strengths. Furthermore, we computed the parameter $\kappa_{\perp}$ for different values of the correlation length $l$ as this value is known only to a small degree of certainty. Our results now largely reconcile the observed values with the theoretical predicted ones as visualized in Fig. 2. For correlation lengths between 5 and $100 \mathrm{pc}$, the theoretical value and the observed one agree within a factor of 2. This agreement is quite good given the difficulties in the observational measurements of diffusion coefficients for Cosmic Ray electrons from radio continuum observations in galaxies. We can confidentially exclude correlation lengths smaller than $1 \mathrm{pc}$, which would result in too small perpendicular diffusion coefficients.

We have done this study for only one galaxy, because so far measurements particularly of the vertical diffusion coefficient are very sparse in galaxies. However, the agreement of the observations with the theoretical prediction lends to some degree support both to the theoretical description of Cosmic Ray diffusion as described in this paper and to the observational attempts to measure it from the Cosmic Ray electron distribution.

Support by the Natural Sciences and Engineering Research Council (NSERC) of Canada is acknowledged. $V . H$. is funded as postdoctoral research assistant by the UK's Science and Technology Facilities Council (STFC).

REFERENCES 
Beck, R. \& Krause, M. 2005, AN, 326, 414

Beck, R. 2007, EAS Publ. Ser., 23, 19

Beck, R. 2012, Springer Space Science Reviews, 166, 215

Breitschwerdt, D., de Avillez, M.A., Feige, J. \& Dettbarn, C. 2012, AN, 333, 486

Cho, J., Lazarian, A., \& Vishniac, E. T., ApJ, 564, 291, 2002

Dosch, A., Shalchi, A., \& Weinhorst, B. 2009, Advances in Space Research, 44, 1326

Goldreich, P., \& Sridhar, S. 1995, ApJ, 438, 763

Haverkorn, M. \& Heesen, V. 2012, Springer Space Science Review, 166, 133

Heesen, V., Beck, R., Krause, M. \& Dettmar, R.-J. 2009a, A\&A, 494,563

Heesen, V., Krause, M., Beck, R. \& Dettmar, R.-J. 2009b, A\&A, 506,1123

Heesen, V., Beck, R., Krause, M., \& Dettmar, R.-J. 2011, A\&A, 535, A79

Krause, M. 2009, Magnetic Fields in the Universe II, ed. A. Esquivel, RevMexAA, 36, 25

Jokipii, J. R., Kóta, J., \& Giacalone, J. 1993, Geophysical Research Letters, 20, 1759

Jones, F. C., Jokipii, J. R., \& Baring, M. G. 1998, ApJ, 509, 238

Matthaeus, W. H., Gray, P. C., Pontius, D. H. Jr., \& Bieber, J. W. 1995, Phys. Rev. Lett., 75, 2136

Qin, G., Matthaeus, W. H. \& Bieber, J. W. 2002, Geophys. Res. Lett., 29

Reynolds, S.P., Gaensler, B.M. \& Fabrizio, B. 2012, Springer Space Science Review, 166, 231
Schlickeiser, R. 2002, Cosmic Ray Astrophysics (Springer, Heidelberg)

Shalchi, A. \& Schlickeiser, R. 2005, ApJ, 626, L97

Shalchi, A., Bieber, J. W., Matthaeus, W. H. \& Schlickeiser, R., ApJ, 642, 230, 2006.

Shalchi, A. 2009, Nonlinear Cosmic Ray Diffusion Theories, Astrophysics and Space Science Library, Vol. 362 (Berlin: Springer)

Shalchi, A., Škoda, T., Tautz, R. C. \& Schlickeiser, R. 2009, Phys. Rev. D, 80, 023012

Shalchi, A. 2010, ApJ, 720, L127

Shalchi, A., Büsching, I., Lazarian, A., \& Schlickeiser, R. Perpendicular Diffusion of Cosmic Rays for a Goldreich-Sridhar Spectrum, The Astrophysical Journal, 725, 2117, 2010.

Shalchi, A. \& Büsching, I. 2010, ApJ, 725, 2110

Soida, M.; Krause, M.; Dettmar, R.-J.; Urbanik, M., A\&A, 531, A127

Swordy, S. P., Müller, D., Meyer, P., L'Heureux, J. \& Grunsfeld, J. M., 1990, ApJ 349, 625

Tautz, R. C. \& Shalchi, A. 2011, ApJ, 735, 92

Tautz, R. C. \& Shalchi, A. 2012, submitted to Journal of Geophysical Research

Westmoquette, M. S., Smith, L. J., \& Gallagher, III, J. S. 2011, MNRAS, 414,3719 